\title{
Surgical Outcomes of Single-Port Laparoscopic Surgery Compared With Conventional Laparoscopic Surgery for Appendiceal Mucinous Neoplasm
}

\author{
In Jun Yang, Minseol Seo, Heung-Kwon Oh, Jeehye Lee, Jung Wook Suh, Duck-Woo Kim, Sung-Bum Kang \\ Department of Surgery, Seoul National University Bundang Hospital, Seongnam, Korea
}

Purpose: This study aimed to evaluate the safety and feasibility of single-port laparoscopic surgery (SLS) for appendiceal mucinous neoplasm (AMN) when compared with conventional laparoscopic surgery (CLS).

Methods: This retrospective study enrolled patients who underwent surgery for AMN between July 2014 and June 2020 at Seoul National University Bundang Hospital. Patient demographics, surgical data, pathology, hospital stay, postoperative morbidity, and follow-up data were extracted from electronic records for analysis.

Results: We enrolled 18 patients who underwent SLS and 22 who underwent CLS. The SLS group included patients who underwent partial cecectomy (14 patients), ileocecectomy (3 patients), and right hemicolectomy (1 patient). The CLS group included patients who underwent appendectomy (4 patients), partial cecectomy (11 patients), ileocecectomy (5 patients), and right hemicolectomy ( 2 patients). Operation type was not significantly different between groups $(\mathrm{P}=0.213)$. No patient required open surgery in the SLS group in contrast to the CLS group (13.6\%; $\mathrm{P}=0.238)$. The operative time tended to be shorter in the SLS group than the CLS group (median [interquartile range]: 52.5 minutes [40-65.2 minutes] and 60 minutes [ $40-120$ minutes], respectively; $\mathrm{P}=0.251$ ). Morbidity was $5.5 \%$ in the SLS group and $9.0 \%$ in the CLS group $(\mathrm{P}=0.692)$. Surgical margins were clear in all cases. The median duration of postoperative hospital stay was 2.0 and 4.0 days in the SLS and CLS groups, respectively $(\mathrm{P}=0.013)$. No recurrence occurred in either group during follow-up. Conclusion: This study indicates that SLS is a safe and feasible surgical approach for AMN.

Keywords: Appendiceal neoplasms; Mucocele; Laparoscopy

\section{INTRODUCTION}

Appendiceal mucinous neoplasm (AMN) is an obstructive dilation of the appendiceal lumen with mucus accumulation most commonly caused by epithelial proliferation. Far less commonly, the cause of AMN is inflammatory or obstructive, including appendicitis and obstruction by a fecalith. The condition is rare,

Received: Sep 3, 2020 - Revised: Nov 2, 2020 - Accepted: Nov 8, 2020 Correspondence to: Heung-Kwon Oh, M.D.

Department of Surgery, Seoul National University Bundang Hospital, 166 Gumi-ro, Bundang-gu, Seongnam 13620, Korea

Tel: +82-31-787-7105, Fax: +82-31-787-4055

E-mail:crsohk@gmail.com

ORCID: https://orcid.org/0000-0002-8066-2367

(C) 2021 The Korean Society of Coloproctology

This is an open-access article distributed under the terms of the Creative Commons Attribution NonCommercial License (https://creativecommons.org/licenses/by-nc/4.0) which permits unrestricted noncommercial use, distribution, and reproduction in any medium, provided the original work is properly cited. with an incidence of $0.2 \%$ to $0.3 \%$ of total appendectomies [1]. There are many pathological classifications for AMN, with the World Health Organization classification being the most widely used [2]. This classification system suggests 3 subtypes of AMN; mucinous adenoma, low-grade AMN, and mucinous adenocarcinoma. In terms of clinical presentation, AMN can cause abdominal pain, abdominal masses, and weight loss, although only $50 \%$ of patients with the condition are symptomatic [3]. Most AMN lesions are incidentally detected during computed tomography (CT), ultrasonography, or endoscopy. The treatment for AMN is surgical resection of the lesion with safe margins, which must be performed without rupture as dissemination of the mucin in the peritoneal cavity can lead to pseudomyxoma peritonei $[3,4]$. The choice of surgical approach is based on the characteristics of the lesion such as size, depth, location, and histopathology $[5,6]$. Recently, the procedures of appendectomy, ileocecectomy, and right hemicolectomy have all become possible through laparoscopic 
surgery, which is a widely chosen approach in various surgical fields because it is associated with reduced pain and size of the surgical wound [7]. There have been several case reports of the successful application of single-port laparoscopic surgery (SLS) for appendectomies, cholecystectomies, and gynecologic procedures [8-10]. In addition, SLS has recently become an alternative option to conventional laparoscopic surgery (CLS) because it minimizes patients' pain and improves cosmetic results as the surgery is performed through a single port at the umbilicus [11]. However, there is still controversy as to whether SLS is safe for patients with AMN because it is a technically challenging procedure. There have only been a few case reports of the use of SLS for resection of AMN $[12,13]$. Therefore, this study aimed to assess the feasibility and safety of this approach in patients with AMN.

\section{METHODS}

\section{Patients}

This retrospective study enrolled patients with AMN without perforation who underwent SLS or CLS at our hospital between July 2014 and June 2020 . We retrospectively reviewed data from electronic patient records that included patient demographics, presurgical laboratory results, surgical data, tumor size and histology, postoperative duration of hospital stay, duration of follow-up, mortality, morbidity, and recurrence. All patients had been clinically diagnosed with AMN, and tumors had been initially detected during radiological examination or colonoscopy as a regular check-up procedure or for abdominal pain. All data were anonymous. The Institutional Review Board of our hospital approved this study before commencement of data collection and analysis (No. B-2001-586-105), and the need for informed consent was waived.
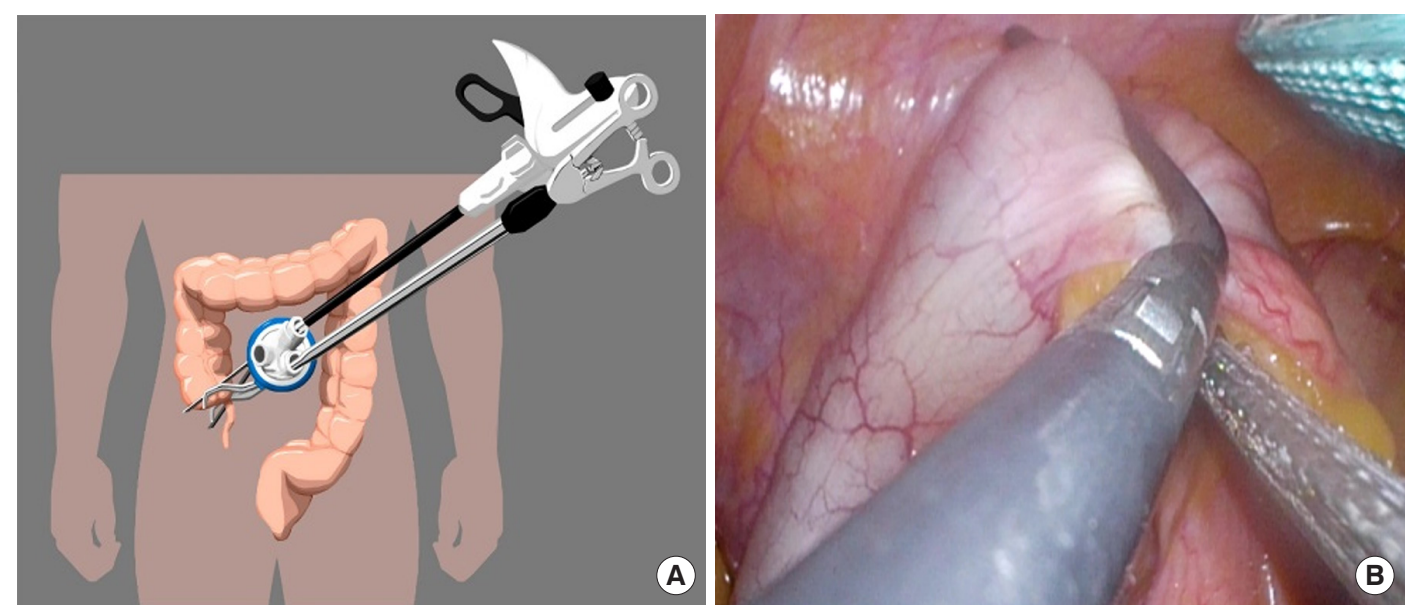

Fig. 1. Laparoscopic surgery for appendiceal mucinous neoplasm. (A) Illustration of single-port laparoscopic surgery using a stapler and endo-Satinsky clamp. (B) Intraoperative photograph showing the tumor being pushed to the distal side toward the appendiceal tip using a 5-mm endo-Satinsky clamp so that the tumor margin remained clear.

\section{Surgical methods}

The surgical approach was selected based on preoperative findings including laboratory and radiologic data, such as tumor characteristics and expected surgical challenges. Surgery was performed under general anesthesia. The American Society of Anesthesiologists (ASA) physical status (PS) classification was evaluated by the anesthetist, and individual anesthetic care was performed based on the ASA PS classification. Patients were maintained in the Trendelenburg position and upright during surgery. The surgeon stood on the left side of the patient while the patient remained supine. A single, vertical $2.5-\mathrm{cm}$ transumbilical incision was made to enter the peritoneal cavity, and a single port (Glove port, Nelis, Bucheon, Korea) was inserted through the incision. The abdomen was insufflated to $12 \mathrm{mmHg}$ with carbon dioxide. Then, a 10-mm laparoscopic camera (Laparo-Thoraco Videoscope [3D], Olympus, Tokyo, Japan) was inserted through the port. Partial cecectomy was performed when the AMN was close to the orifice of the appendix or when there was a high probability that the AMN would remain in the resection margin by simple appendectomy. Ileocecectomy and right hemicolectomy were performed when tumor invasion was broad or the tumor could not be resected by partial cecectomy. After identifying the appendix, the mesoappendix was transected from the appendiceal base to the cecum using monopolar cautery or advanced energy devices. Then, the appendiceal artery was ligated. The AMN was pushed to the pelvic side using a 5-mm endo-Satinsky clamp (B. Braun, Tuttlingen, Germany) to ensure a clear tumor margin (Fig. 1). Using a laparoscopic linear stapler (Echelon Flex, $60 \mathrm{~mm}$; Ethicon Endo-Surgery, Cincinnati, OH, USA), the cecum was resected and extracted through the umbilical incision. Finally, the port was removed, and the wound was closed layer by layer. 


\section{Statistical analyses}

Categorical variables are presented as number (percentage) and continuous variables as median (interquartile range). We used the Mann-Whitney U-test to compare continuous variables and Fisher exact test or the chi-square test to compare categorical variables. A P-value of $<0.05$ was considered statistically significant. All statistical analyses were performed using IBM SPSS Statistics ver. 22 (IBM Corp., Armonk, NY, USA).

\section{RESULTS}

A total of 53 patients underwent surgery for an AMN during the study period. Among these, 13 were excluded because they were diagnosed with other diseases such as endometriosis. Finally, 40 patients were enrolled for analysis, 18 of whom had undergone SLS, whereas 22 had undergone CLS. Regarding their AMN classification, 39 patients had low-grade AMN, and 1 had high-grade AMN. The mean age of the patients was 60.8 years (range, 37-81 years), and the mean body mass index was $24.5 \mathrm{~kg} / \mathrm{m}^{2}$. The median operation time was 60.0 minutes (range, 39.2-112.5 minutes). Partial cecectomy was performed most frequently (in 25 cases), followed by ileocecectomy in 8 , appendectomy in 4 , and right hemicolectomy in 3 cases. The median tumor size was 6.5 $\mathrm{cm}$, and the median length of hospital stay was 3.5 days. The median follow-up period was 14 months (range, 0-51 months).

Baseline characteristics in relation to the type of surgery (SLS or CLS) are presented in Table 1 . The mean age and body mass index were higher among patients who had undergone CLS com-

Table 1. Baseline characteristics of the study patients

\begin{tabular}{lccc}
\hline Characteristic & SLS & CLS & P-value \\
\hline No. of patients & 18 & 22 & \\
Age $(\mathrm{yr})$ & $60.6(37-81)$ & $61.0(39-80)$ & 0.989 \\
Sex & & & 0.436 \\
$\quad$ Male & $6(33.3)$ & $10(45.5)$ & \\
$\quad$ Female & $12(66.7)$ & $12(54.5)$ & \\
BMI (kg/m²) & $23.9(20.6-26.7)$ & $25.0(22.9-26.3)$ & 0.411 \\
ASA PS classification & & & 0.615 \\
I & $8(44.4)$ & $7(31.8)$ & \\
II & $9(50.0)$ & $12(54.5)$ & \\
III & $1(5.6)$ & $0(0)$ & \\
IV & $0(0)$ & $0(0)$ & \\
Smoking & $3(16.7)$ & $1(4.5)$ & 0.310 \\
Operation history & $5(27.8)$ & $9(40.9)$ & 0.386 \\
\hline
\end{tabular}

Values are presented as number only, mean (range), number (\%), or median (interquartile range).

SLS, single-port laparoscopic surgery; CLS, conventional laparoscopic surgery; BMI, body mass index; ASA, American Society of Anesthesiologists; PS, physical status. pared to those who had undergone SLS, although there was no statistical difference. For 3 patients in the CLS group, there were no ASA PS classification data (Table 1). The type of surgery and surgical details are summarized in Table 2. The median operative time of the CLS group tended to be longer than that of the SLS group, whereas the median postoperative hospital stay was significantly shorter in the SLS group $(\mathrm{P}=0.013)$. The surgical margins were clear in all patients, and there were no cases of conversion to CLS or open surgery in the SLS group, which was lower than the rate of conversion to open surgery in the CLS group. The median estimated blood loss was significantly lower in the SLS group than in the CLS group $(\mathrm{P}=0.008)$, and mortality was $0 \%$ in both groups. Morbidity was not significantly different between groups. No recurrence, such as pseudomyxoma peritonei, occurred in either group.

\section{DISCUSSION}

To the best of our knowledge, this is the first study to compare the surgical outcomes of SLS with those of CLS for the treatment of AMN. This study revealed that the operative time, morbidity, and mortality of SLS are not significantly different from those of CLS. However, the estimated blood loss and postoperative hospital stay were significantly lower for SLS, possibly because right colectomy and ileocecetomy were performed more frequently in the CLS group than in the SLS group. Furthermore, patients requiring open surgery were more frequent in the CLS group, because 2 out of 3 had a history of previous surgery.

Table 2. Surgical outcomes

\begin{tabular}{lccc}
\hline Variable & SLS $(\mathrm{n}=18)$ & CLS $(\mathrm{n}=22)$ & P-value \\
\hline Operative time (min) & $52.5(40.0-65.2)$ & $60.0(40.0-120.0)$ & 0.251 \\
Operation type & & & 0.213 \\
$\quad$ Appendectomy & $0(0)$ & $4(18.2)$ & \\
Partial cecectomy & $14(77.8)$ & $11(50.0)$ & \\
Ileocecectomy & $3(16.7)$ & $5(22.7)$ & \\
Right hemicolectomy & $1(5.6)$ & $2(9.1)$ & \\
EBL (mL) & $3.0(0.0-30.0)$ & $30.0(12.5-30.0)$ & $0.008^{*}$ \\
Open conversion & $0(0)$ & $3(13.6)$ & 0.238 \\
Tumor size (cm) & $5.2(4.2-6.5)$ & $7.9(5.5-9.2)$ & 0.079 \\
Postoperative hospital stay & $2.0(2.0-3.0)$ & $4.0(3.0-5.75)$ & $0.013^{*}$ \\
30-Day morbidity & $1(5.5)$ & $2(9.0)$ & 0.692 \\
30-Day mortality & $0(0)$ & $0(0)$ & \\
Recurrence & $0(0)$ & $0(0)$ & \\
\hline
\end{tabular}

Values are presented as median (interquartile range) or number (\%).

SLS, single-port laparoscopic surgery; CLS, conventional laparoscopic surgery; $\mathrm{EBL}$, estimated blood loss.

${ }^{*} \mathrm{P}<0.05$. 
The AMN is rarely encountered in clinical practice because it occurs when there is an abnormal accumulation of mucin in the appendix causing abnormal distension after obstruction by a fecalith or appendicolith [14]. An AMN is typically observed as an incidental finding when $\mathrm{CT}$ or magnetic resonance imaging (MRI) is performed for other reasons. The appearance of AMN on CT is a tubular lesion or round cyst with low attenuation and enhanced wall of the appendix. In MRI, the appearance of an $\mathrm{AMN}$ is a hyperintense, cystic pericecal mass on T2-weighted images. On T1-weighted images, the mass is usually hypointense, but with variable intensity depending on the mucin content [2]. Surgical resection with negative margins is the standard treatment, and AMN without signs of perforation or peritoneal invasion can be safely treated with appendectomy alone [3]. According to previous studies, open surgery is safe because it reduces the possibility of peritoneal seeding during specimen extraction [4]. However, a recent study revealed that laparoscopic surgery is a safe approach for the treatment of AMN [15].

Laparoscopic surgery has been applied to various surgical fields and is becoming a popular technique in many surgical fields, with SLS being widely applied to simple surgeries such as appendectomy and cholecystectomy $[8,9]$. Furthermore, the use of SLS for treating cancer is increasing [16], and the technique is attracting the attention of patients and physicians because of its advantages in terms of improved postoperative pain and cosmetic outcomes [8]. However, the application of SLS for AMN is controversial because of the technical challenges involved, particularly, the concern of mucin spillage. In the present study, we did not observe mucin spillage in any of the patients who underwent SLS, and the operative time was short for this procedure. Therefore, our results suggest that SLS may be safer and less technically difficult than CLS for the treatment of AMN. The indication for SLS in the treatment of AMN has not been established yet. Our data suggest that SLS has better outcomes than CLS if the tumor is near the cecal base. The endo-Santisky clamp, which can be used in SLS because the single port is larger than a conventional port, helps in resecting the tumor by clipping the cecum vertically and pushing the tumor toward the tip of the appendix. A stapler can then be inserted through the single port and the cecum resected safely.

The present study has some limitations which should be acknowledged. First, this was a retrospective, single-center study with a small number of enrolled patients. We recognize that the results may not be generalizable to a significantly larger, more variable population. In addition, the results are inherently subject to bias because of the retrospective nature of the study. Therefore, prospective, multicenter studies with larger sample sizes are needed to confirm our findings. Second, the follow-up period was not long enough to evaluate long-term outcomes.

In conclusion, we found that SLS is a safe and feasible surgical approach for AMN.

\section{CONFLICT OF INTEREST}

No potential conflict of interest relevant to this article was reported.

\section{ACKNOWLEDGMENTS}

We would like to express our sincere gratitude to Ji Hwan Kim for his illustration.

\section{REFERENCES}

1. Smeenk RM, van Velthuysen ML, Verwaal VJ, Zoetmulder FA. Appendiceal neoplasms and pseudomyxoma peritonei: a population based study. Eur J Surg Oncol 2008;34:196-201.

2. Van Hooser A, Williams TR, Myers DT. Mucinous appendiceal neoplasms: pathologic classification, clinical implications, imaging spectrum and mimics. Abdom Radiol (NY) 2018;43:2913-22.

3. Stocchi L, Wolff BG, Larson DR, Harrington JR. Surgical treatment of appendiceal mucocele. Arch Surg 2003;138:585-9.

4. González Moreno S, Shmookler BM, Sugarbaker PH. Appendiceal mucocele: contraindication to laparoscopic appendectomy. Surg Endosc 1998;12:1177-9.

5. Glasgow SC, Gaertner W, Stewart D, Davids J, Alavi K, Paquette IM, et al. The American society of colon and rectal surgeons, clinical practice guidelines for the management of appendiceal neoplasms. Dis Colon Rectum 2019;62:1425-38.

6. Kelly KJ. Management of appendix cancer. Clin Colon Rectal Surg 2015;28:247-55.

7. Kim TK, Park JH, Kim JY, Kim BC, Kang BM, Min SK, et al. Safety and feasibility of laparoscopic surgery for appendiceal mucocele: a multicenter study. Surg Endosc 2018;32:4408-14.

8. Cho MS, Min BS, Hong YK, Lee WJ. Single-site versus conventional laparoscopic appendectomy: comparison of short-term operative outcomes. Surg Endosc 2011;25:36-40.

9. Suh SW, Choi Y, Han HS, Yoon YS, Cho JY, Choi YS, et al. Solo single-incision laparoscopic cholecystectomy: a safe substitute for conventional laparoscopic cholecystectomy. ANZ J Surg 2019;89: 900-4.

10. Xie W, Cao D, Yang J, Yu M, Shen K, Zhao L. Single-port vs multiport laparoscopic hysterectomy: a meta-analysis of randomized controlled trials. J Minim Invasive Gynecol 2016;23:1049-56.

11. Duza G, Davrieux CF, Palermo M, Khiangte E, Azfar M, Rizvi SA, et al. Conventional laparoscopic appendectomy versus singleport laparoscopic appendectomy, a multicenter randomized control trial: a feasible and safe alternative to standard laparoscopy. J Laparoendosc Adv Surg Tech A 2019;29:1577-84.

12. Park KB, Park JS, Choi GS, Kim HJ, Park SY, Ryuk JP, et al. Single-incision laparoscopic surgery for appendiceal mucoceles: safety and feasibility in a series of 16 consecutive cases. J Korean Soc Coloproctol 2011;27:287-92.

13. Ishibashi K, Okada N, Ohsawa T, Kumamoto K, Haga N, Ishida H. 
A simple and safe technique for performing single-port laparoscopic resection of appendiceal mucocele. Tech Coloproctol 2011; 15:341-3.

14. Louis TH, Felter DF. Mucocele of the appendix. Proc (Bayl Univ Med Cent) 2014;27:33-4.

15. Park KJ, Choi HJ, Kim SH. Laparoscopic approach to mucocele of appendiceal mucinous cystadenoma: feasibility and short-term outcomes in 24 consecutive cases. Surg Endosc 2015;29:3179-83.

16. Diana M, Dhumane P, Cahill RA, Mortensen N, Leroy J, Marescaux J. Minimal invasive single-site surgery in colorectal procedures: current state of the art. J Minim Access Surg 2011;7:52-60. 